Chen, Q., Glicsman, L., Lin, J., and Scott, A. 2007. “Sustainable urban housing in China,” Journal of

Harbin Institute of Technology (New Series), 14s, 6-9.

\title{
Sustainable Urban Housing in China
}

\author{
Qingyan CHEN ${ }^{1}$, Leon GLICKSMAN ${ }^{2}$, Juintow LIN ${ }^{3}$, and Andrew SCOTT ${ }^{2}$
}

(1. School of Mechanical Engineering, Purdue University, 585 Purdue Mall, West Lafayette, IN 47907, USA, Email: yanchen@purdue.edu. 2. Department of Architecture, Massachusetts Institute of Technology, 77 Massachusetts Ave., Cambridge, MA 02139, USA, 3. Fox Lin Inc., 2432 Ocean Ave., Venice, CA 90291, USA)

\begin{abstract}
As China is becoming more urbanized, about 3.5 million units of housing are built every year. It is very important to develop sustainable urban housing in China. A team of researchers and developers from the USA and China have worked together to develop several housing projects in Beijing, Shanghai, and Shenzhen. These designs are neither "hi-tech" solutions nor "super-advanced" technologies. Instead, the team provided a socially and technically sustainable development in a high-density setting with a central design goal of achieving symbiotic and environmentally sensitive relationships. The relationships preserve Chinese culture, promote social interactions among the residents, and reduce energy consumptions by decreasing heat loss and maximizing solar gain in the winter and by shading the sun and allowing natural ventilation in the summer. This paper outlines a design example that uses state-of-the-art design tools to achieve the goals of sustainable design.
\end{abstract}

Key words: Energy, Environment, Culture, Design, Technology, Case Studies

\section{Introduction}

The overarching goal of sustainable development is to preserve and improve the global environment for future generations. The population growth for Chinese cities is 16 to 18 million and about 3.5 million units of apartments being built annually. China's contribution to sustainability is particularly important because of its accelerated growth rate and improving rank in the world economy, and can therefore articulate the early combined benefits of rapid growth with sustainable design.

We have worked together with several universities and developers in China to explore design, technology, and implementation of environmentally responsive urban housing in three large Chinese cities with different climates, Beijing, Shanghai and Shenzhen. Recently, a book ${ }^{[1]}$ has been published as an outcome of the effort. This paper presents a part of the results from the book.

\section{Research Methods}

Two typologies of buildings dominate the urban landscape of new construction in China; these include low-rise walk-up buildings that are arranged as long building masses (Fig. 1a), and high-rise towers that have little relationship to the environment and ground plane (Fig. 1b). Recent low-rise housing has changed a little bit to enhance security and service, but there are no substantial changes in terms of resident interactions and energy use by the buildings.

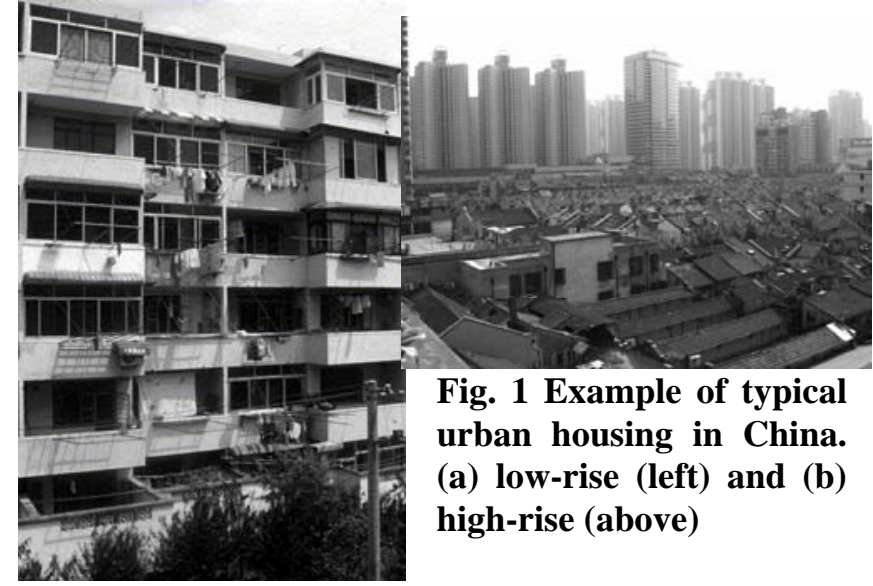

We have developed a series of aspiring development projects that could point towards a better way of designing with environmental responsibility and social interactions in mind. We did not seek to impose new "hi-tech" solutions or "super-advanced" technologies. Conversely, the attitude that underpins our work has been to rediscover and interpret the traditions of Chinese housing. The discovery and interpretation are in terms of typologies that incorporate social structures and the means by which buildings have traditionally been responsive to working with the climatic forces. For example, courtyard housing forms of siheyuan (Fig. 2) for centuries provided for the needs of the sustainable community from which we can absorb the quintessence.

Contemporary housing has to meet many needs beyond those of the vernacular due to higher densities, better social conditions, stricter building codes and standards, and the increased mobility of the industrialized society - in contrast to those types from 
the agrarian society of the past. The sustainability of housing is interpreted in multiple modes: social, technological, climatic, and urban. Some are related to performance for better efficiency or economy of resources and energy while others are about quality of life and the ability of people to live together without alienation.

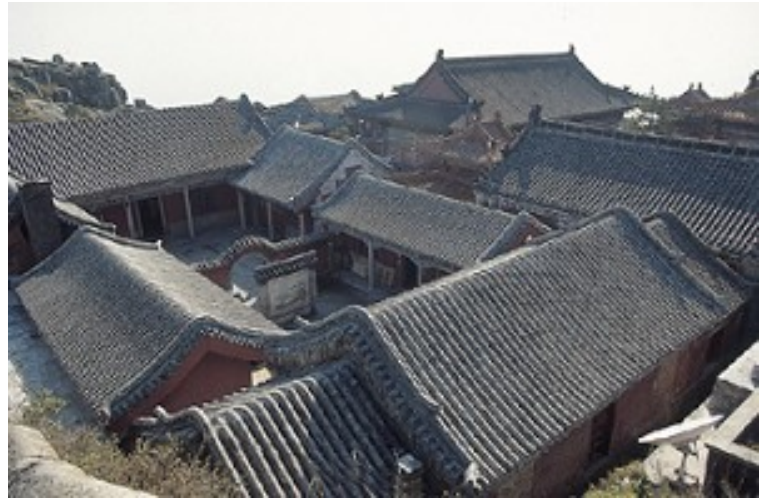

Fig. 2. A typical courtyard housing - Siheyuan

Thus, on one hand, our strategies were to develop plans that could preserve culture, encourage social interactions, and build a sense of community. On the other hand, we sought design solutions where the landscape can be more ecologically responsive in relation to water conservation and retention and to carbon dioxide absorption; and where the housing can be far more energy efficient. The energy efficient design is to conserve energy by better insulation and reduced infiltration, to maximize the use of solar energy for daylighting and winter heating, and to use solar shading and natural ventilation for summer cooling. However, rather than developing a design that might contain a large palette of available "sustainability techniques," we sought to develop an understanding of those concepts and technologies that would be most effective; and therefore would make greatest sense for each project.

The investigations focused upon the careful design of individual building interiors and exteriors, as well as building groupings. Design concepts were developed through state-of-the-art modeling tools of sun, wind, and daylighting for energy and the environment. The housing projects selected were representative of units that a substantial number of urban residents could afford, not only a few millionaires. The focus of this work was on the combined architectural and technical design of sustainable buildings.

\section{Case Studies}

To illustrate how we developed sustainable urban housing in China, this section use Huilongguan project in Beijing shown in Fig. 3 as an example. The project is to develop $89,000 \mathrm{~m}^{2}$ housing with an emphasis on ecological, environmental, and economic sustainability.

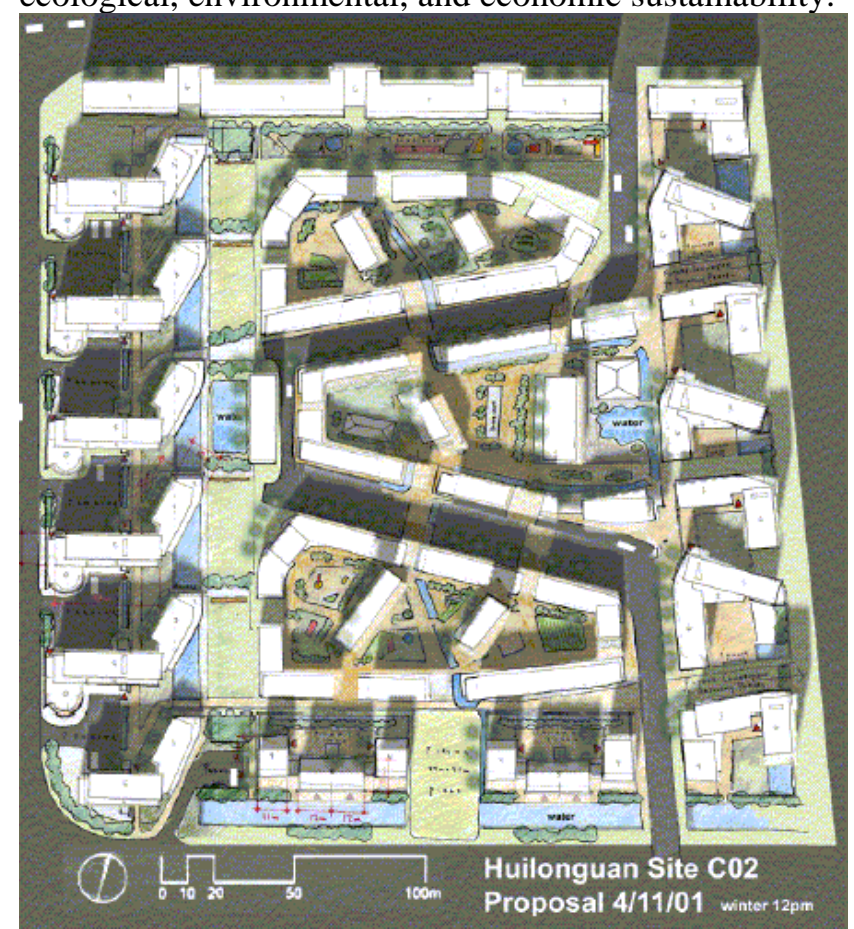

Fig. 3. Aerial view of Huilongguan project scheme

\subsection{Socially sustainable design}

A primary effort for the project was to provide a socially sustainable development in a high-density setting with a central design goal of achieving symbiotic and environmentally sensitive relationships between the individual dwelling and communal areas of the development. Many high-rise residential developments in the area neglect a sociological perspective characterized by community spaces with poorly articulated relationships between public and private space. The Chinese culture has long term promoted social interactions through courtyard in Beijing. Modern Chinese are also very interested in protecting their privacy while enjoying more freedom. Residential developments must address the sociological issues related to numbers of unrelated persons living in close proximity. We sought to accomplish this by providing public spaces of various scales and types for social interactions, as well as comfortable private living areas.

Public areas between major paths or in the central site area were designed to be places where all residents of the housing development would feel welcome. The 
buildings were arranged in groups by site area, each with its own distinct community space. A community space for the site as a whole was provided, as well as neighborhood areas geared towards use by residents of each individual building group. Numerous "private spaces" were created by means of small green areas partially secluded from the roads and the pedestrian pathway traffic. Gardens, open green spaces, groves of trees, and a water canal were interspersed through the site to provide a connection to nature throughout. The large grass-covered areas, while both aesthetic and recreational, also added to the overall site surface permeability and helped to combat carbon dioxide emissions.

\subsection{Technically sustainable design}

For the Beijing climate, with cold winters and hot summers, the objective was to simultaneously decrease heat loss by increasing insulation and reducing infiltration; maximize solar gain through windows in the winter months; while to shade sun through windows and allow natural ventilation for cooling in the summer months, as illustrated in Fig. 4.

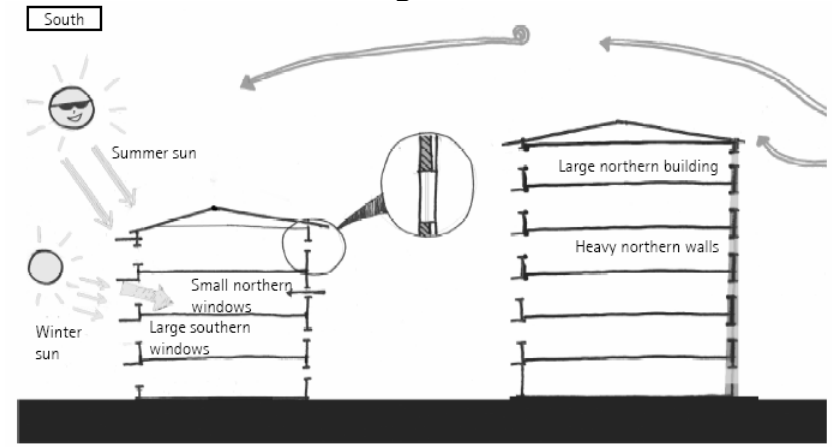

Fig. 4. Sustainable technologies selected.

To minimize heat loss in winter, the most effective measures are to use insulation and to reduce infiltration. By using $50 \mathrm{~mm}$ of foam insulation, the heat loss from the buildings can be reduced by $60 \%$, while the increase in construction costs is minimal.

The infiltration rate for any given building is primarily a function of construction standards and the wind speed outside of the building. Placing taller buildings on the winter upwind (north) side of the project could reduce the infiltration rate by reducing the local wind speed around adjacent buildings as shown in Fig. 5 that was obtained by using a computational fluid dynamics (CFD) program. Reducing the outdoor airspeed by $50 \%$ could reduce the infiltration rate by about $50 \%$ or more.

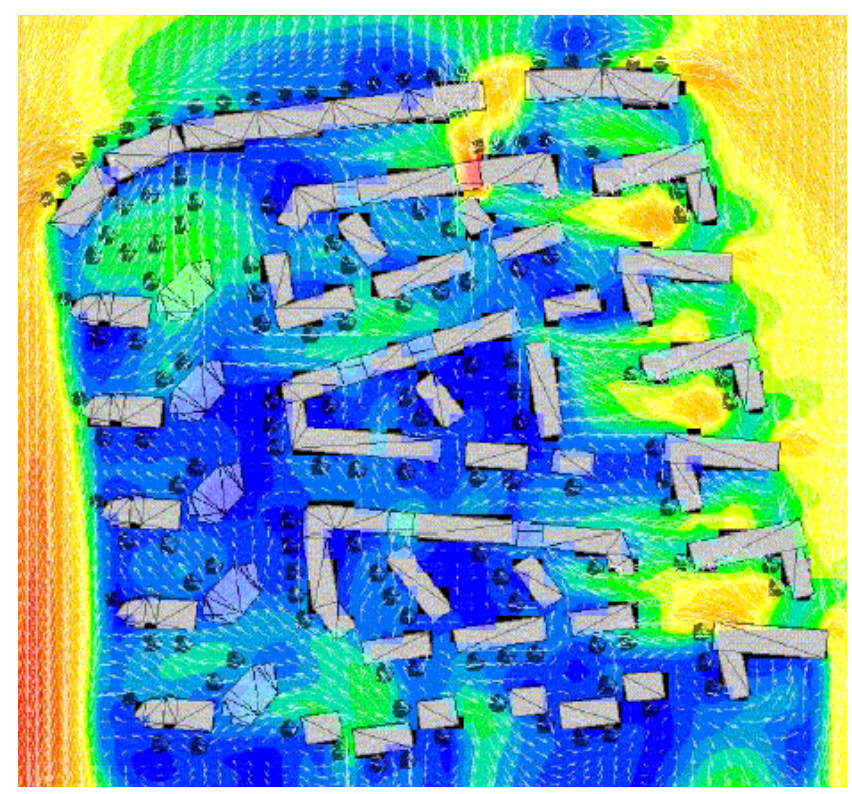

Fig. 5. Wind distribution in winter on the site, blue low velocity, yellow - medium velocity and red - high velocity

We assumed 0.5 and 2.0 ACH infiltration during heating season for low and high construction quality, respectively, and two different insulation levels, $10 \mathrm{~mm}$ and $50 \mathrm{~mm}$ of foam in the walls and the roof. It was determined that there was a substantial increase in energy efficiency when moving from the low quality construction and low insulated building to the high quality construction and highly insulated building. The seasonal heating energy was reduced from $161 \mathrm{kWh} / \mathrm{m}^{2}$ to $35 \mathrm{kWh} / \mathrm{m}^{2}$.

The building code also requires two-hour sun in any apartment on December 21. Thus, we used a lighting computer program to analyze the sun availability. As shown in Fig. 3, our design can satisfy the code requirement and ensure the sun is available for each apartment.

With the sun, Fig. 6 shows increasing the area of double-glazed windows on the south decreased heating requirements, while doing the same for single-glazed windows had the opposite effect because the windows conduct heat too readily. The calculation was with a window area of $20 \%$ for all north, east, and west walls. Our design used concrete floor slab, representing a large thermal mass, to store solar energy during the day for release later at night. This would utilize excess solar energy at day that otherwise would not be harnessed, and could help to reduce peak heating loads late at night. 


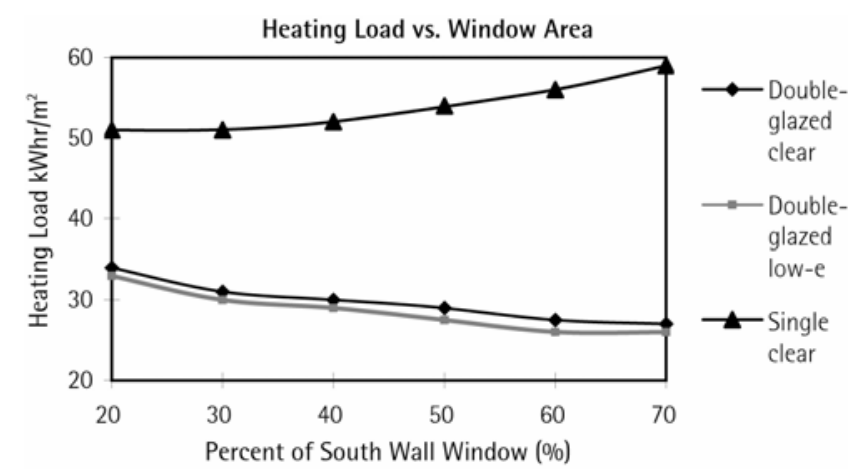

Fig. 5 Heating with increasing southern window area

Note that an increase of window area caused an increase of the cooling load, unless the windows are carefully shaded to reduce direct solar energy in the summer. Therefore, it was recommended that windows be shaded. Fig. 6 shows the shading effect for August 21 with an overhang depth equal to $50 \%$ of window height and a fin depth equal to $50 \%$ of window width. The shading effect is very evidence in reducing summer cooling energy from $2.1 \mathrm{kWh} / \mathrm{m}^{2}$ window area to 0.25 $\mathrm{kWh} / \mathrm{m}^{2}$.

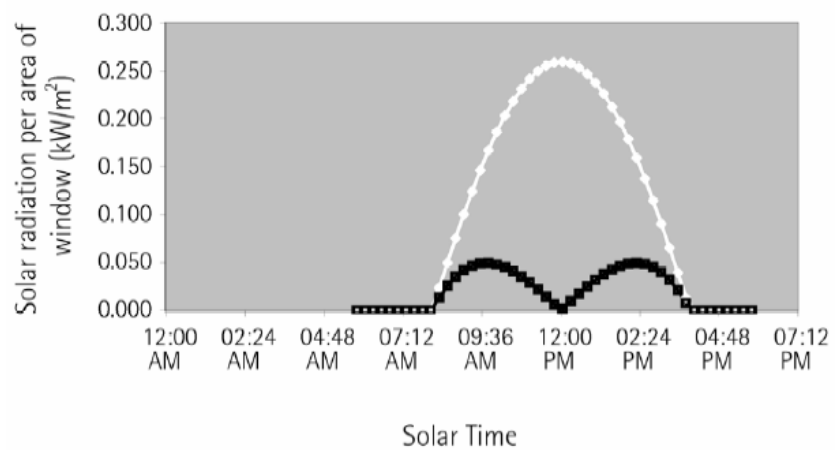

Fig. 6 Solar radiation through a south facing window with (black line) and without shading (white line)

We have also analyzed the possibility to use natural ventilation for summer cooling when the wind is dominantly from the south. With the design, the analysis showed air movement even within the central cluster spaces at an average $0.5 \mathrm{~m} / \mathrm{s}$. The majority of the site saw some air movement only slightly lower than the original wind at $2 \mathrm{~m} / \mathrm{s}$ from the south. With nighttime cooling and the thermal mass from the floor structure, the indoor air temperature in such apartments will not be higher than $32^{\circ} \mathrm{C}$ in the summer when the outdoor air temperature can be over $38^{\circ} \mathrm{C}$.

\section{Discussion}

The sustainable design achieved here used state-ofthe-arts design tools, such as computer programs for daylighting, energy, and flow analysis. Some of the programs are very sophisticated. Small and medium design firms may not have the skills or could not afford such tools.

Sustainability is not on the top list of home owners' mind in China. It is difficult to sell real sustainability concept presented in this paper to the Chinese developers and home owners. Chinese government should provide incentives through its energy pricing policy.

\section{Conclusions}

This paper presents a sustainable concept for urban housing in China. A socially and technically sustainable development in a high-density setting should preserve Chinese culture, promote social interactions among the residents, and reduce energy consumptions by decreasing heat loss and maximizing solar gain in the winter and by shading the sun and by allowing natural ventilation in the summer.

\section{Acknowledgements}

We would like to thank many former graduate students from MIT who contributed to the results presented in the paper. Other contributors include Tsinghua University, Tongji University, and Beijing Tianhong Group. This study was financially supported by the Alliance for Global Sustainability. We would like also to thank Springer for its permission to reprint some of the results from the book "Sustainable Urban Housing in China”.

\section{References:}

[1] GLICKSMAN LR and LIN J. 2006 Sustainable Urban Housing in China, Springer. 